\title{
THE APRIL MEETING IN NEW YORK
}

The six hundred sixty-fourth meeting of the American Mathematical Society was held at the Americana Hotel in New York on April $2-5,1969$. All sessions were held in public rooms of the hotel. About 502 persons attended, including about 360 members of the Society.

By invitation of the Committee to Select Hour Speakers for Eastern Sectional Meetings, there were four addresses. Professor David Shale of the University of Pennsylvania spoke on Friday morning on Some probabilistic ideas in mathematical physics. He was introduced by Professor Mark Kac. Professor Cathleen Morawetz of New York University spoke on Friday afternoon on Energy flow: wave motion and geometrical optics. She was introduced by Professor Lipman Bers. Professor Katsumi Nomizu of Brown University spoke on Saturday morning on Differential geometry of complex hypersurfaces. He was introduced by Professor Richard S. Palais. Professor William Browder spoke on Saturday afternoon on Invariants of manifolds. He was introduced by Professor Samuel Eilenberg.

There were sixteen sessions, including two late sessions, for 119 contributed papers during the mornings and afternoons of Friday and Saturday. These sessions were chaired by Paul Axt, Stephen L. Bloom, Ethan Bolker, David K. Cohoon, Lawrence J. Corwin, Barnett W. Glickfeld, Jonathan L. Gross, Anthony W. Hager, Carl Kallina, Boo-Sang Lee, Wallace S. Martindale, III, Raymond A. Mayer, Jr., Peter Perkins, Stewart M. Robinson, Edwin H. Rogers, William H. Ruckle, Choy-Tak Taam, Clifton Whyburn.

On Wednesday and Thursday there was a symposium on Mathematical aspects of electrical network theory, sponsored by the AMSSIAM Committee on Applied Mathematics, and supported by the Air Force Office of Scientific Research and the U. S. Army Research Office (Durham). The Organizing Committee consisted of Dr. W. A. Blackwell, Dr. Frank Branin, Dr. Robert Brayton, Professor Frank Harary, and Professor Herbert S. Wilf (chairman). Hour addresses were given by Professor J. W. T. Youngs, University of California, Santa Cruz; Dr. Robert Brayton, IBM Corporation; Dr. D. C. Youla, Brooklyn Polytechnic Institute; and Professor R. J. Duffin, Carnegie-Mellon University. There were also sessions for shorter papers.

The Council met on April 4, 1969 in the Versailles Terrace of the Americana of New York at 5:00 P.M. 
The Secretary reported that the following persons represented the Society at various functions as noted:

E. E. Floyd at the One Hundred and Fiftieth Anniversary of the University of Virginia

Herbert C. Kranzer at the inauguration of Allyn P. Robinson as President of Dowling College

Earl Lavender at the inauguration of John Olin Eidson as President of Georgia Southern College

E. Baylis Shanks at the inauguration of M. G. Scarlett as President of Middle Tennessee State University

Trevor Evans at the inauguration of Vivian Wilson Henderson as President of Clark College

Clyde T. McCormick at the inauguration of Robert S. Eckley as President of Illinois Wesleyan University

Eugene H. Lehman, Jr. at the inauguration of John X. Jamrich as President of Northern Michigan University

Harold Hanes at the inauguration of John J. Pruis as President of Ball State University

Donald W. Western at the inauguration of William H. Duncan as President of Millersville State College

Arthur Kaufman at the inauguration of Herbert Schueler as President of Richmond College

Miguel A. Guitart at the inauguration of Kermit Alonzo Johnson as President of Alabama College

George M. Stratopoulos at the inauguration of Glen Laird Taggart as President of Utah State University

Russell J. Michel at the Southern Illinois University Centennial Period Charter Day Convocation

R. D. Boswell, Jr. at the inauguration of John Torben Bernhard as President of Western Illinois University.

The following Presidential appointments have been made:

Committee to Select Hour Speakers for Western Sectional Meetings: Roger Lyndon, replacing L. deBranges who resigned.

Nominating Committee: A. H. Taub, Chairman, R. S. Palais, K. M. Hoffman, D. C. Spencer, P. E. Thomas.

Committee on Expository Writing: J. A. Dieudonné, P. R. Halmos, M. M. Schiffer, N. E. Steenrod, Chairman.

Committee to Nominate Winner of Cole Prize: E. R. Kolchin, M. A. Rosenlicht, John T. Tate, Chairman.

Committee on the Welfare of Mathematicians and Mathematics 
Students in Involuntary Service: Creighton Buck, Chairman, Chandler Davis, J. L. Kelley.

The Council received reports of Executive Committee Business-byMail. The following persons were elected to ordinary membership:

Bellamy, David P., University of Delaware;

Benzinger, Leonora A., George Mason College, University of Virginia;

Bissonnette, Donald K., University of Arizona;

Breiter, Thomas A., Wisconsin State University, La Crosse;

Bruning, Linda Mae, University of Nebraska;

Chipman, John S., University of Minnesota;

Christensen, Howard M., University of Colorado;

Cole, Raymond E., Hewitt Associates, Los Angeles, California;

Collison, Joseph E., Grumman Aircraft Corp., Bethpage, New York;

Coon, Dorothy Trautman, Otterbein College;

Curtis, Edward B., Massachusetts Institute of Technology;

Daghestani, Burhandeen A. Z., North Texas State University;

Dartsch, Robert C., Evergreen Park High School, Illinois;

De Bruijn, Johannes, McGill University;

Donahue, William W., New College;

Fernandez, Cesar L., University of Chile, Santiago, Chile;

Fuller, Roy J., University of Arkansas;

Garrett, Bert Dee, University of Alabama;

Gotlieb, Calvin C., University of Toronto;

Haines, David C., Iowa State University;

Hardy, Kenneth, Carleton University;

Hare, William R. Jr., Clemson University;

Harvey, Douglas P., Wayne State University;

Helton, John W., Suny at Stony Brook;

Hepp, Klaus, Swiss Federal Institute of Technology, Zurich, Switzerland;

Honkomp, Robert J., Xavier University;

Hsu, Ta Chean, Christian Brothers College;

Hubbuck, John R., Manchester University, Manchester, England;

Judge, David J., University College, Dublin, England;

Kahaner, David K., Los Alamos Scientific Laboratory, New Mexico;

Kislinger, John D., General Electric Company, Syracuse, New York;

Kizer, Wallace Lee, Baptist College at Charleston;

Kulshrestha, Prem K., Louisiana State University in New Orleans;

Laranjeira, Manuel, Comissao De Energia Nuclear, Lisbon, Portugal;

Laskar, Renu C., Clemson University;

Lawson, Reginald O., University of Colorado;

Leech, Robert B., University of Nebraska;

Lee, Kotik K., Syracuse University;

Lippman, Gary E., University of California, Riverside;

Lloyd, Kirce W., RCA Service Company, Cocoa Beach, Florida;

Manougian, Manoug N., University of South Florida;

Markham, Thomas L., University of South Carolina;

Markowsky, George, Harvard University;

Massey, Winston L., University of Chattanooga;

McGowan, Francis S., Grumman Aircraft Corp., Bethpage, New York; 
McMorris, Fred R., University of Wisconsin, Milwaukee;

Miller, Gary G., University of Victoria;

Morris, Cecil A., Virginia Polytechnic Institute;

Mufarrij, Michel M., American University of Beirut, Beirut, Lebanon;

Mullen, James A., Raytheon Company, Waltham, Mass.;

Nardi, V., University of Padova, Padova, Italy;

Newman, James M., Florida Atlantic University;

Olivares, Alberto E., Consulting Engineer, Los Caobos, Caracas;

Padberg, Sister Harriet Ann, Maryville College;

Papanicohaou, George C., New York University at Courant;

Penney, Carol W., University of Georgia;

Proctor, E. Langley Jr., Bendix Field Engineering Corporation, Maryland;

Rahn, James R., Gordon College;

Rhemtulla, Akbar H., University of Alberta;

Rice, Bart F., Louisiana State University;

Riebe, Norman W., Arizona Western College;

Savariraj, G. A., St. Josephs College, Tiruchirapalli, India;

Scalisi, Ignatius P., Northeastern University;

Shook, Thurston W., Ohio State University;

Sternbach, Leonard P., Ohio State University;

Sulton, Arnold L. Jr., Computing and Software Incorporated, Maryland;

Tannenwald, Ronald M., Southeastern Massachusetts Technological Institute;

Toralballa, Leopoldo V., New York University;

Ukena, Ann E., Washburn University of Topeka;

Urner, Ronald M., Air Force Institute of Technology;

Van Winter, Clasine, University of Kentucky;

Wang, Fuyao, Colorado State College;

Weiss, Leonard, Brown University;

Wheatley, Patrick Owen, University of Houston;

Wilde, Carroll O., Naval Postgraduate School;

Wilkinson, James B., Erskine College;

Williams, Louis F. Jr., University of Florida;

The following persons were elected to membership as nominees of institutional members:

University of Alberta: R. Anantharaman, William M. Freed, S. V. Modak, Joanne V.

Peeples, M. S. Phul, L. Bruce Richmond, Brian Douglas Warrack.

Amherst College: James G. Mauldon, Edwin Mershon Wolf.

Andrews University: Bruce A. Kesselring, Martha Ann Luss, Barbara Ann Swope.

Angelo State College: Johnny Matt Bailey, Noel D. Evans.

Arizona Stale University: James E. Gehrmann, Merilyn F. George, Judith L. Gersting,

Edwin Joseph Hewett.

University of Arkansas: J. D. Hansard, David R. Simmons, Joseph C. Warndof.

Auburn University: James Austin French, J. C. Propes, James R. Sidbury.

Bates College: Earl M. L. Beard, Judith M. Isaacson, Richard W. Sampson.

Boston College: James E. Cavanaugh, Karen J. Schroeder.

Brandeis University: John H. Baker, Tamar Burak, Miriam H. Hellman, Albert M.

Leisinger, Daniela Liebowitz, Phillip C. Lynch, Margaret S. Menzin, Ralph Reid,

David G. Schaeffer, Glenn D. Sproul, Stuart Turner.

University of Bridgeport: Elizabeth A. Smith.

Brown University: Richard F. Basener, George W. H. Blundall, Jr., Robert Joseph 
Bond, Joseph A. Erbacher, Lawrence R. Ernst, John H. Ewing, Steve Galovich, Robert M. Hardt.

California Institute of Technology: Haines J. Boyle, William G. Bridges, Peter G. Dodds, J. Robert Henderson, John P. Lindal, William C. Lydord, Thomas J. Noyes, K. D. Stroyan.

California State College at Fullerton: Linda Christine Leigh, Lee E. Williamson.

California State College at Long Beach: J. A. Hammervold, John K. Wood.

University of California at Davis: Neal Phelps Carpenter, Herbert L. Holden, Elmo Moore, Jr., Edmund Francis Schramko, Carver S. Simmons.

University of California at Irvine: Martin E. Walter.

University of California at Los Angeles: Nguyen Anh, Charles E. Antoniak, Taeigeun Cho, Patrick Barry Eberlein, Paul H. Galyean, Bernard A. Glassman, Jerrold M. Gold, David B. Gould, John Gregory, Robert D. Hall, Richard Irvin Harris, Franklin T. Iha, Frank Kontrovich, Young Koan Kwon, Peter Alan Laszlo, I. H. Lin, T. R. McCutcheon, Tyrone L. McMurrey, Ysahaswini Deval Mittal, Mohsen Pazirandeh, John Piepenbrink, Frederic M. Pollack, Joel L. Weiner.

Calvin College: John J. Bajema.

Carnegie Mellon University: Charles A. Cheney, Robert P. Daley, Benjamin D. Haytock, Robert N. Moll, Enrique J. Risler, G. E. Trapp, Jr., Robert A. Valosik, Nathaniel R. Withers.

Colorado State University: Marian S. Brown, J. Penelope Crothers.

University of Colorado: Roxanne M. Byrne, Kyong Soo Chung, Richard H. Elderkin, Steven K. Ingram, James A. Leise, Thomas J. Norris, Ronald Shonkwiler, Susan J. Zimmerman.

University of Connecticut: William O. Dixon, Mary J. Ellien, Gary H. Ford, Peter T. Laseau, J. Mannarino.

Cornell University: Donald Alvin Alton, William H. Cutler, Alan H. Durfee, Ross Georgehan, S. Halperin, Lawrence A. Harris, Richard Andrew Holley, Hui Hsiung Kuo, Kenneth I. Mandelberg, L. Taylor Ollmann, Youichi Saigusa, Martin M. Sternstein, John Brendan Sullivan.

Dartmouth College: R. I. Gisselquist.

University of Dayton: Charlotte E. McConn, Harlod L. Schoen.

Drexel Institute of Technology: A. J. Herr, Mark P. Saks, Julianne Souchek.

Duke University: Malcolm G. Lane.

East Carolina University: Etang Chen, Lokenath Debnath, Katharine W. Hodgin.

Eastern New Mexico University: Douglas Black, Truman Menefee, Juanita Thompson. Emory and Henry College: Schery Lodter Young.

Fordham University: Robert A. Bagnato, Sister Mary Ann Dirr, Anthony L. Labruzza. Franklin and Marshall College: Roy Goldman.

George Washington University: Barbara Caldwell Brobeck, Hugo D. Junghenn, James D. McPherson.

Georgetown University: Joseph T. Chambers, Jr., P. H. Gould, John E. Kauflin. Georgia Institute of Technology: David L. Brown.

University of Georgia: Ivan Ivansic, James W. Maxwell, Bill E. Swafford.

Harvard University: Lewis Burch Davis, John D. Garberson, Lawrence R. Gonick, Deborah Hughes Hallett, Birger Iverson, Carl Pomerance, S. Ramanan, Barry K. Rosen.

Hofstra University: Douglas S. Brown, Jeanne Ferrante, Stanley Paul Goldberg.

Hope College: John L. Van Iwaarden, John T. Whittle.

Idaho State College: Buddy D. Kolander.

University of Idaho: Richard Dee Barnhart.

University of Illinois at Chicago Circle: David C. Feinstein. 
University of Illinois: Walter E. Beck, Charles H. Bertness, David A. Birnbaum, Marshall L. Cates, Lloyd E. Chambless, Paul E. Cohen, Douglas A. Cope, Janet E. Dorman, Lawrence P. Endo, Joel C. Farrar, Gerald J. Giaccai, Gary L. Hensler, Janice M. Huang, Marvin M. Israel, Edwin H. Kaufman, Jr., John C. Kieffer, Talma O. Leviatan, John C. Lewis, Larry C. Lipskie, Hal Liu, Turg T. Marti, Joseph O. Mercer, Carman B. Miller, Larry J. Morley, Donald H. Pelletier, George B. Purdy, Lonnie J. Roth, Stuart A. Steinberg, John Steing, Ross H. Street, Peter C. Trombi, Jacqueline D. Tulumello, Lakshmi G. Vijaya, Kasturi Viswanath, James J. Woeppel, Harvey E. Wolff.

Institute for Advanced Study: J. Cerf, E. Brien Davies, Koji Doi, Peter Donovan, John A. Gibbs, George F. Haddix, Koichiro Harada, Dennis Johnson, Mitsuyoshi Kato, I. G. MacDonald, Hidehisa Naganuma, M. S. Narasimhan.

Iowa State University: Albert M. Erisman, Daryl R. Fischer, Melvin L. Klasi, Robert W. Neufeld, David O. Oakland, Galen Roger Peters, Dale M. Rognlie, A. L. Ruby.

University of Iowa: Ralph A. Czerwinski, Marjory Jane Johnson, Douglas L. Nelson, R. Richard Summerhill; Jane F. Wells.

Johns Hopkins University: David E. Ansel, Paul Ellner, Ronald N. Goldman, Aomoto Kazuhiko, Timothy King, Suresh M. Moolgavkar, Hisasi Morikawa.

Kansas State University: L. A. Cammack, Garry D. Hart, James P. Hatzenbuhler.

University of Kansas: Tommy K. Teague, Charles I. Voiaw.

Kent State University: L. F. Delattre, Yin Hoang, Tau Ku.

University of Kentucky: Ben C. Brewster.

Knox College: Michael Joseph Goetsch, William A. Schiesser, Ralph Collins Walter.

Lehigh University: Stephen Ray Alpert, Grahame Bennett, Jeffrey C. Bosserman, James R. Herz, Jr., Rudolph Martin Koch, Edward F. Sabotka, Elias H. Toubassi.

Lemoyne College: Linda F. Masters, Harry L. Reeves.

Louisiana State University: Robert W. Yeagy.

McGill University: William J. Anderson.

McMaster University: R. Alan Day, Meera Gupta, Shu Bun Ng.

Macalster College: Charles C. Hanna, Kenneth G. Miller, Judy M. Seppanen.

University of Maine: Sara L. Costa, Alfred Darrow, Alfred Bruce Fant.

Massachusetts Institute of Technology: Jeffrey H. Albert, Alberto Baider, Wendell T. Beyer, Steven L. Blumsack, Robert Jonathan Bobrow, Michael J. Cowen, Thomas A. Fiske, Martin A. Golubitsky, Richard J. Grant, Richard Haberman, Chung Wu Ho, Edmund F. Kelly, John B. Lewis, Arthur Lieberman, A. Mobile, Linda Esther Preiss, Arnold G. Reinhold, Gerald Jacob Roskes, Josef Sukonick, Laurence A. Wolsey.

University of Massachusetts: Keith R. Ferland, Theodore A. Giebutowski, Victor P. Schneider, William Lee Shelden, Jr.

University of Michigan: Marine B. Abrahamse, Jozef Blass, Cynthia B. Brown, David Eugene Cowles, Maurice D. Craig, Joseph K. Cross, Antonio O. Farias, David E. Flesner, John James Hinrichsen, Paul E. Howard, Nance Evelyn King, David Irvin Lawson, Robert Allen Liebler, Erik A. Lippa, James D. McCall, Jr., R. Wayne Patterson, John R. Quine, Jr., Pedro P. Sanchez, Norman L. Schryer, Paul K. Stockmeyer, Manda B. Suryanarayana, Henry Edward Thomas, Jr.

University of Missouri: Massoud M. Awad, Marvin S. Keener, William Ookian Ke, Fred Stroup, Jr., Robert F. Wheeler.

University of Missouri at Kansas City: Todd Kelso, Robert C. Middleton, Larry E. Sherwood.

University of Missouri at Rolla: Thomas S. Angell, Alexander H. Cramer. 
University of Missouri at St. Louis: Esther Yuen.

University of Montana: Robert W. Kopitze, James A. Pollock, Charles W. Schelin.

University of Montreal: Arturo A. L. Sangalli.

Naval Postgraduate School: Franklin Lee Daniels.

University of Nebraska: Walter R. Woodward.

University of Nevada: Earl Cassity, Douglas M. Colbert, Richard Tim Mizner.

University of New Hampshire: Paul L. Estes, Garfield C. Schmidt, Raj K. Singh.

New Mexico State University: A. N. Dabboucy, Jim Olin Howard, Manuel Keepler,

Andrew Y. Schoene, William Andrew Theddford, Sharon Kay Theleman.

New York University: George F. Weinberger.

North Carolina State University at Raleigh: Joby Milo Anthony, Stephen K. Park, Douglas Bodsford Smith, Jr.

University of North Carolina at Chapel Hill: Federico E. Heinz, John M. McVoy, Jack W. Pope, James A. Sawyer, Jr.

University of North Carolina at Charlotte: Lloyd D. Davis, Wanda F. King, Miriam A. Leiva.

University of North Carolina at Greenboro: Patricia Anne Griffin, George D. Joyner, William K. Link, Jr.

University of North Dakota: James L. Elliott, Joseph A. Guzek.

North Texas State University: James Darrell Heath, Carlus E. Lewis, Charles Vonler.

Northern Michigan University: Robert B. Buchanan, Sharon L. McCulley, Gwendolyn C. Neul.

Northwestern University: Kuang Ho Chen, Lawrence S. Evans, Joel C. Gibbons, George L. Grossman, Martin A. Keane, Edwin M. Klein, Andy Roy Magid, Richard J. Maher, Donald K. Mason, Barry C. Peterson, Don D. Porter, Donald C. Raffety, Shmuel Rosset, Zeev Schuss, Gerald B. Silverman, William B. Vesley, Brian Richard Lau.

University of Notre Dame: Paul L. Chabot, Gunther H. Frei, J. R. Senft, Katsnmi Yagi. Ohio University: Esther L. Duncan, Larry Edward Irwin, Lee Parsons.

Old Dominion College: Reinhart Hitz.

University of Oregon: Robert L. Bohac, Michael E. Burke, Mark E. Christie, S. N. Dua, Gary A. Gislason, A. C. Granzin, Mark M. Holte, Douglas A. Parker, David Alan Schoenfeld, Lynn D. Slingerland, Bill Watson.

University of Ottawa: Klaus K. Kanneman, Gerald A. Shea.

Pacific Lutheran University: Robert S. Fisk, Ronald J. Ness, Thomas E. Stuen.

University of Pittsburgh: J. Vincent Moroose, Thomas W. Rishel.

Portland State University: Lawrence C. Ford, Gary Hunter, Jack E. Slingerland.

Princeton University: Craig J. Benham, M. Emin Bozhuyuk, Ibrahim Dibag, Robert M. Ephraim, Harry Felder, III, Gerald B. Folland, Charles N. Friedman, Deborah Louise Goldsmith, Bonnie Gold, Mark Lee Green, Martha R. Katzin, Leonard Lipshitz, John Myron Masley, V. Alan Norton, Marjorie L. Stein, James C. Thorpe.

Purdue University: Jagdish C. Agrawal, Achmad Arifin, Jean Pierre L. Aubin, Anthony P. Blozinski, Juliane F. Bokobza, David C. Buchthal, Gerry L. Chrisman, Thomas Carney, Paul C. Duchateau, Carl F. Eckberg, Thomas G. Hack, Thomas J. Hartz, Arun D. Joshi, Stephen J. Klein, Joseph M. Lambert, George E. Lang, Jr., John J. Loughlin, Ras B. Painaik, Ramachandrachar V. Rao, Jerry Lee Searcy, David L. Shannon, Eileen T. Torney, Michael P. Underwood, Andre Unterberger, Byungho Yoon.

Rensselaer Polytechnic Institute: Edward P. Culp.

University of Rhode Island: Hardwin Blanchard, Donald Girod, Linda C. Hill, Z. R. Manare. 
Rockford College: Donald G. Fuller.

Rutgers University: Eugene M. Gaydos, Zachary Granovetter, Louie M. Mahony. Sacramento State College: William A. Hardy, Ben H. Mulkey, Paraskevi Steinberg.

Saint Marys College of Maryland: Kim Ki Hang Butler.

Saint Olaf College: Sue Martens, Linda A. Ness, Neil J. Omyedt.

San Diego State College: Stanley J. Benkoski, Jr., Robert N. Devich, Stephen R. Johnson.

University of Santa Clara: Charles F. Swart.

University of Saskatchewan at Regina: Norman A. Beirnes, J. W. Bingaman, Carl L. Cunningham.

Simon Fraser University: John T. Baldwin, Herbert S. Gaskill, Gary C. W. Sabin.

South Dakota School of Mines and Technology: Robert N. Higgins, IV, V. Kay Long, Carol G. Myers.

Southern Illinois University at Carbondale: Barry J. Blonde, John R. Haddock.

Southern Methodist University: Terye Bock Dubner, Rebecca C. Howell.

Stanford University: Alfred D. Andrew, John H. Bolstad, Jacob Burbea, Roger Stephen Carne, Donald M. Davis, Paul G. Davis, William Lloyd Davis, John M. Dedinsky, Henry Koch Dewitt, Philip J. Faillace, J. L. Finkelstein, Barbara Rani Fink, Fred Gama Lobo, Herman Ruge Jervell, Daniel F. X. O'Reilly, Michael Perloff, A. H. Schoenfeld, Henry David Shapiro, Gary G. Ventep, John Victor Wagner, Jr., Leonard D. Weisberg, Robert S. Winternitz, Robert A. Wolfe.

State University of New York College: William C. Bosch, Alice Joy Bourne, Leroy R. Lundin.

State University of New York at Binghamton: Edward W. Odell.

State University of New York at Buffalo: Renganathan Aravamudhan, George Theodore Georgantas, L. N. Gupta, Jose B. Mason, Rajagopalan Neela, Saraswathi Subbiah.

Stephen F. Austin State College: Kent Macdougall, David H. Major, Donald R. O. Quinn.

Stevens Institute of Technology: Roger S. Pinkham.

Suffolk University: Richard Lee Parris.

Syracuse University: Said S. Abu Zahra, Karl A. Beres, Margaret W. Groman, Donald G. Hazlewood, Edward K. Kwak, Charles Dwight Lahr, Chin Yeang Lim, Alexander J. Lindsey, Edmund A. Memmott, Shantilal N. Shah, Carl B. Shepardson.

University of Tennessee: John W. Baker, Charles D. Bass, Juliana Dowell, Robert E. Funderlic, John O. Kork, J. R. Ridenhour, Robert Jennings Simpson, Joseph E. Wagner, Martha Thomas West.

Texas A \& $M$ University: Alton R. Crawley, Carter G. Lyons, Thomas F. McCabe. Texas Christian University: Michael J. Gray.

Texas Technological College: Gerald V. McWilliams, James L. Poirot, Robert L. Sartain.

University of Texas: Robert B. Anderson, Richard N. Brodie, David F. Phillips.

University of Toledo: J. V. Cano, Raymond A. Heitger.

Tulane University of Louisiana: William A. Greene, Sheila R. O'Donnell.

University of Utah: James M. Briggs, A. George Dors, Richard Franke, Frank G. Lether, Gregory Morris Nielson, Stephen Kent Parker.

Vassar College: Carolyn F. Colburn, Armen G. Fisher, Dennis L. Micham.

University of Virginia: William C. Chewning, Jr., Andrew J. Galiley, Gary C. Hamrick, Joseph F. Kent, Robert Edward Lewand, Russell J. Rowlett, Roy L. Smith.

Virginia Polytechnic Institute: James M. Boyte, Douglas E. Cameron, Ted W. Goodman. 
Washington and Lee University: Robert B. Brownell, James J. Pollard.

Washington State University: Dennis K. Burke, John R. Rabung.

Washington University: Humberto R. Alagia, Eduardo H. C. Cattani, Yao Pi Chen, Robert E. Clapp, Aroldo G. Kaplan.

University of Washington: Iracema Martin Bund, Eugene F. Cornelius, Jr., James A.

Draper, Frank D. Farmer, Ruth E. Little, David J. Lutzer, Kelly Denis McKen-

non, Lynn McLinden, Walter Michaelis, Robert H. Overton, Andrew Bad Hwa Wang.

Wayne Stale University: Robert M. Cantor, Martin N. Fealk, Alise P. Lenss.

Wells College: Stephen Bancroft.

Wesleyan University: William P. Berlinghoff, Cecilia H. Brook, Jason Gait, Wesley J. Orser, Michael E. Paul.

Western Washington State College: Stanley H. Jensen, Margaret A. Johnston, Peter V. McCarthy.

Wichita State University: Alan R. Elcrat, Dean K. Kukral, Ronald P. Teemly.

College of William and Mary: Larry Keith Barker, Robert C. Ward.

Williams College: Henry M. Walker.

University of Wisconsin at Madison: Martin W. Bartelt, Ellis A. Bauman, Miro Bemda, William Boos, Sylvia R. Cohen, Leroy W. Cooper, Donald S. Coram, Warren Wilder Esty, Samuel R. Filippone, Daniel M. Fitzgerald, Marsha F. Forman, Raymond A. Garcia, Bradford S. Gile, Frederic Gooding, Jr., Madelyn Fern Hartman, Theodore J. Hartman, Stephen P. Hayward, Elizabeth H. Johnson, Nancy M. Kilty, Hendrik J. Kuiper, Sinan Kunt, James J. Kuzmanovich, Gary L. Lachmund, David C. Leung, Tina Leslie Levy, Shlomo Libesking, Kuang Chi Liu, Norman J. Loomer, Joyce R. Malleck, Edward J. Mayland, Jr., Robert G. McKean, John Thomas Montgomery, Douglass B. Morris, M. E. Nadel, John P. Norback, Mitchell S. Nussbaum, Michael Olinick, David Alex Oppenheim, John D. Prange, David S. Protas, Ira N. Rosenholtz, Andre E. Samson, Philip B. Sheldon, Frederic W. Shultz, David A. Stegenga, Kenneth Ray Stephenson, Dean E. Stowers, Robert A. Straughn, Jr., Joseph Anthony Troccolo, Dallas Eugene Webster, Esther Weinstock, Ronald Joseph Werhnyak, C. Edwin Wilson, Joel Zinn.

University of Wisconsin at Milwaukee: Paul D. Humke, James K. Kasum, Viktor G. Kurotscka.

Wofford College: Grace M. Y. C. Hu.

Yale University: Bruce N. Allison, Ian D. Brown, Robert S. Cahn, Robert G. Chipkin, Malcolm A. Coulter, Carol W. Coven, Daniel M. Fendel, Dante M. Giarrusso, Reginald C. Haines, Stella M. Hammond, Donal J. Hurley, Stephen C. King, Paul Ponomarev, Michel L. Racine, Arnold Mark Sedaca, Richard Walter Sharpe, Elizabeth Wall.

York University: Joseph D. Horton, Neil A. Weiss.

The following individuals were elected to membership in accordance with reciprocity agreements with the various mathematical organizations named:

Union Matematica Argentina: Felipe Joaquin Zo.

Australian Mathematical Society: Bruce D. Craven, R. C. Hughes, Hans Lausch, James M. McPherson.

Osterreichische Mathematische Gellschraft: Wilfried H. Nobauer.

Societe Mathematique de Belgique: Alfred Edmond Warrinnier.

Societe Mathematique de France: Emilio Isla. 
Deutsche Mathematiker Vereinigung: Ernst Boenecke, Lothar O. Collatz, Hans F. Gunzler, Erich Ossa.

Gesellschraft für Angewandte Mathematik und Mechanik: Bernhard H. Korte, Peter Weidner.

The London Mathematical Society: Derek J. S. Robinson, Geoffrey V. Wood.

Indian Mathematical Society: Robert P. Burn, Peng Yee Lee, Brajabanhoo Misra, Hariharaier Subramanian.

Israel Mathematical Union: Morel M. Marcus.

Unione Mathematica Italiana: Bruno Forte, Giuseppe Geymonat.

Mathematical Society of Japan: Mamoru Mimura, Kiyoshi Noshiro, Kunio Oguchi, Igari Satoru, Kano Takeshi, Kaneko Tetuo.

Wiskundig Genootschap te Amsterdam: Hendrik S. DeSnoo.

Svenska Mathematikersamfundet Gesellschaft: Bjorn O. Textorius.

Schweizerische Mathematische Gesellschaft: Bruno P. Zwahlen.

The following institutions were elected to institutional membership:

Bowling Green State University, Bowling Green, Ohio

Concordia College, Moorehead, Minnesota

Newark College of Engineering, Newark, New Jersey

California State College, Dominguez Hills, California

Eastern Oregon College, LaGrande, Oregon

Herbert H. Lehman College of City University of New York, Bronx, New York

College de Sainte Marie, Montreal, Canada

It was announced that the Editorial Committees of the Proceedings and of the Transactions have decided to require an abstract, a descriptive title, AMS subject classification numbers, and key words and phrases for articles submitted after July 1, 1969. [Subsequently, the Editorial Committee of the Bulletin also agreed to require the last three items.]

The Council considered the following resolution:

B. Whereas the American Mathematical Society encourages all persons interested in mathematical research to be members of the Society and whereas these members hold a wide variety of political and social views and have been welcomed to membership without regard to these views, resolved that the Society shall not attempt to speak with one voice for the membership on political and social issues not of direct professional concern and shall adhere closely to the purpose stated in its Articles of Incorporation of "furtherance of the interests of mathematical scholarship and research."

After extended discussion the resolution was unanimously approved, with one abstention, for presentation to the membership for a referendum by mail with favorable recommendation of the Council.

The Council then turned to the consideration of five resolutions introduced at the Business Meeting of the Seventy-Fifth Annual Meeting in New Orleans in January 1969. The resolutions are published in the report of that meeting in this Bulletin, vol. 75, p. 480. 
Acting with the powers conferred in the By-laws, Article IV, Section 8 , first and last paragraphs, the Council agreed, by a vote of 29 to 1 , to present the Resolutions 1 through 5 to the membership for a referendum by mail, with the accompanying statement that the Council recommends a vote against each of the resolutions as a consequence of its recommendation for Resolution $B$.

Although neither the motion on Resolution B nor that on Resolutions 1 through 5 specified a data for the referendum, the tenor of the discussion called for a referendum before the close of the academic year with a report of the results at the August Council and Business Meetings.

The Council considered a limitation on the number of contributed papers to be accepted at the Seventy-Sixth Annual Meeting in Miami in January 1970. They passed a motion that the first seven hundred and twenty acceptable papers be placed on the program in the customary manner (i.e., sorted into sessions by subject and presented during the usual daylight hours) and that any additional papers be scheduled for evening sessions in order of their appearance.

The Council approved a meeting in Madison on April 14-18, 1970, with contributed papers and invited addresses on April 17-18 and possibly a symposium in part or all of the interval April 14-16. The proposed Symposium topic, approved by the Council, is Representation Theory of Finite Groups and Related Topics, with Irving Reiner as Chairman of the Organizing Committee and Editor.

The issue of the location of future meetings in the West again came under discussion. The Council passed a motion that the meeting of April 1971 in the West be held in the city of Chicago, preferably at one of the universities.

Assistant Treasurer Murray Protter presented the financial statement and supplemental schedules for the calendar year 1968, as prepared by the auditors Haskins and Sells. An abbreviated version will be published in the September issue of the Bulletin.

The Council elected Professor Fred Brauer to replace Professor Wasow during his temporary absence from his duties as Editor of the Proceedings.

The Council adjourned at 11:00 P.M.

Everett Pitcher

Secretary

LEONARD GillmaN

Associate Secretary

Bethlehem, Pennsylvania

ROCHESTER, NEW YORK 